Ann. Biol. anim. Bioch. Biophys., I972, 12 (2), 43I-345.

\title{
ESSAI DE PURIFICATION DE LA LIPASE DU TISSU INTERCAECAL DE LA TRUITE (SALMO GAIRDNERI RICH.)
}

\author{
C. LEKGER \\ Station de Recherches de Nutrition, \\ Centre national de Recherches zootechniques, I. N.R. A., \\ 78 - Jouy-en-Josas
}

La Truite possède un pancréas dont les cellules sont dispersées (LAGUesse, I 894) dans un tissu adipo-conjonctif, le tissu intercaecal.BRockerhoFf (I966) avait supposé l'existence d'une activité lipasique au niveau de ce tissu. Nous avons montré dans un précédent article (LÉGER et al., I970) que la mesure in vitro de cette activité était possible et que l'enzyme responsable de cette activité présentait des caractéristiques cinétiques différentes chez des animaux préalablement acclimatés à la température de $10^{\circ} \mathrm{C}$ ou à celle de $20^{\circ} \mathrm{C}$. Ces résultats nous ont conduit à penser que ces modifications étaient le reflet d'une adaptation de la lipase à la température d'acclimatation de la Truite. Nous avons alors envisagé la mise au point d'une méthode de purification de l'enzyme, dans le but de rechercher si ce phénomène était dú à l'existence d'enzymes différentes (isoenzymes) ou d'une même enzyme de différentes conformations. Nous présentons ici nos résultats sur les premiers stades de la purification.

Les activités lipasiques sont mesurées par titrage en continu à pH constant (DesNuelLe, Constantin et BALDY, I955). Le substrat utilisé dans la réaction enzymatique est la fraction triglycéridique de l'huile d'olive obtenue après passage de l'huile sur une colonne de Florisil, l'élution des triglycérides étant effectuée à l'aide d'un mélange hexane-oxyde d'éthyle 24/1 $(\mathrm{v} / \mathrm{v})$. Les teneurs en protéines des échantillons sont évaluées par la technique colorimétrique de Lowny et al. (I95I).

\section{I. - PRÉPARATION D'UN LYOPHIIISAT \\ A FAIBLE ACTIVITÉ LIPASIQUE (LFAL)}

Les truites (1) sont sacrifiées par rupture des vertèbres cervicales. La portion de tube digestif (environ $8 \mathrm{~cm}$ de longueur) sur laquelle débouchent les caecums pyloriques est prélevée, plongée aussitôt dans l'azote liquide et broyée à l'aide d'un hachoir à viande (type Scharfen). Le pro-

(1) Les truites pèsent entre $150 \mathrm{~g}$ et $200 \mathrm{~g}$. 
duit broyé est ensuite lyophilisé. Il est indispensable d'effectuer une lyophilisation à ce stade de l'opération : la délipidation - par des solvants organiques - d'échantillons humides dénature la lipase de façon complète et irréversible.

Les résidus lyophilisés contiennent 65 p. xoo de lipides et présentent une activité spécifique de $0,6 \mathrm{UL} / \mathrm{mg} \mathrm{(}{ }^{(1)}$ de protéines.

\section{II. - PRÉPARATION DE LA POUDRE DÉGRAISSÉE (E)}

La délipidation quasi complète des résidus lyophilisés est effectuée à $0^{\circ} \mathrm{C}$ à l'aide de différents mélanges solvants d'après une technique dérivée de celle de VERGER et al. (1969).

Pour $160 \mathrm{~g}$ de LFAL, nous opérons de la façon suivante : le LFAL est broyé dans $500 \mathrm{ml}$ de solvant pendant $4 \times 30$ secondes à l'aide d'un mixeur à lame rotative de type Turmix au maximum de sa puissance, puis le solvant est filtré sous vide sur papier Whatman $n^{0} \mathbf{I}$; le gâteau ainsi obtenu est repris pour le traitement suivant. L'ordre et le nombre des traitements, ainsi que les solvants utilisés, sont portés dans le tableau suivant :

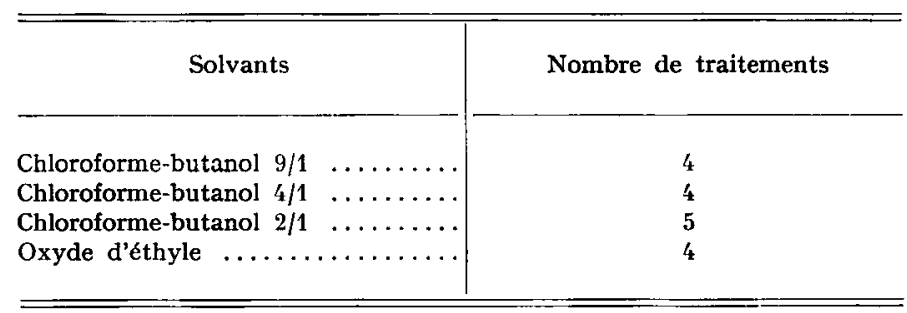

Après le dernier traitement à l'oxyde d'éthyle, la poudre sèche contient moins de I p. roo en poids de lipides saponifiables et $99 \mathrm{p}$. Ioo de protéines. Elle est conservée à $-20^{\circ} \mathrm{C}$; son activité spécifique est de $0,45 \mathrm{UL} / \mathrm{mg}$ de protéines.

\section{III. - PRÉPARATION D'UN LYOPHILISAT A ACTIVI'TÉ LIPASIQUE ÉLEVÉE (LALE)}

Les opérations sont effectuées à $0^{\circ} \mathrm{C}$. La poudre dégraissée est mise en suspension dans le tampon Tris : $0, \mathrm{I} \mathrm{M}\left(\mathrm{pH}_{7,5} ; \mathrm{I}=0,08\right)$ à la concentration protéique finale de 2 p. 100. Après avoir été agitée 2 heures, la suspension est centrifugée $50 \mathrm{mn}$ à $35000 \times 3$ environ. Le surnageant est additionné d'une quantité de sulfate d'ammonium correspondant à $60 \mathrm{p}$. roo de la saturation en sel à $0^{\circ} \mathrm{C}$ (fig. I). Après agitation, la solution est centrifugée $60 \mathrm{mn}$ à $35000 \times 3$. Le culot obtenu est dissous dans une quantité minimale de $\mathrm{NaCl} 0,4 \mathrm{M}$ (inférieure à $5 \mathrm{ml}$ ). Cette solution est placée sur une colonne de Séphadex $\mathrm{G}$ 1oo $(2,5 \mathrm{~cm} \times 40 \mathrm{~cm})$ préalablement équilibrée dans $\mathrm{NaCl} 0,4 \mathrm{M}$ et dont le débit est maintenu à $\mathrm{I} 2 \mathrm{ml} / \mathrm{heure}$ environ. Dans ces conditions, le pic d'activité lipasique est élué immédiatement après le volume d'exclusion de la colonne (cf. fig. 2). Les fractions à activité lipasique sont lyophilisées après dialyse contre l'eau. L'activité spécifique est de ${ }_{5} \mathrm{UL} / \mathrm{mg}$ de protéines.

(1) Unité lipase = I $\mu$ équiv, acide libéré par minute. 


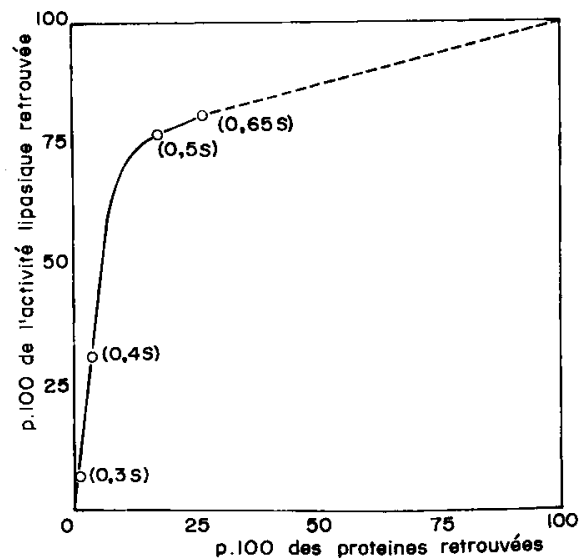

FIG. I. - Courbe de précipitation de la lipase par le sulfate d'ammonium

En abscisse, sont portés les pourcentages de protéines précipitées par rapport aux protéines totales; en ordonnée, sont portés les pourcentages de lipase précipitée pour chaque concentration en sulfate d'ammonium. Cette concentration est exprimée, entre parenthèses, en fraction de saturation.

TABLEAU I

Différentes étapes de la purification

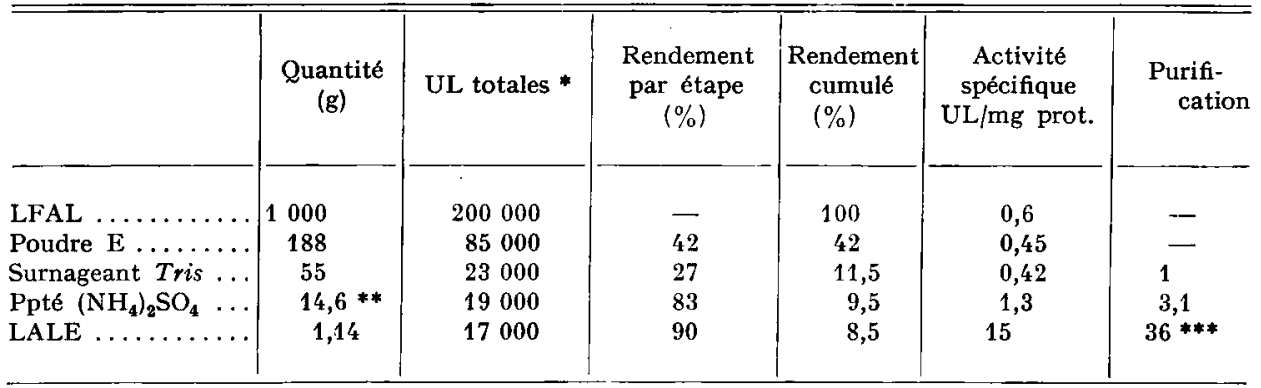

* UL totales mesurées à $\mathrm{pH} 8,7$ et $25^{\circ} \mathrm{C}$.

** Signalons que cet extrait présente des activités trypsique et chymotrypsique spécifiques respectivement de 23 et $80 \mathrm{U} / \mathrm{mg}$ de protéines et ne présente pas d'activité amylasique.

*** La purification finale est de 75 , et non de 36 , si nous exprimons l'activité spécifique de LFAL en UL par mg de lyophilisat entier. 
Le tableau I donne les caractéristiques de chaque étape de la purification.

La figure 2 fait apparaître un déplacement du pic d'élution de la lipase, lorsque la force ionique du milieu croît. Ce phénomène est mis à profit pour atteindre un taux élevé de purification. Il nous suggère, en outre, deux hypothèses : ou bien la lipase pourrait être adsorbée sur des agrégats de poids moléculaires élevés, ou bien des sous-unités de la lipase seraient

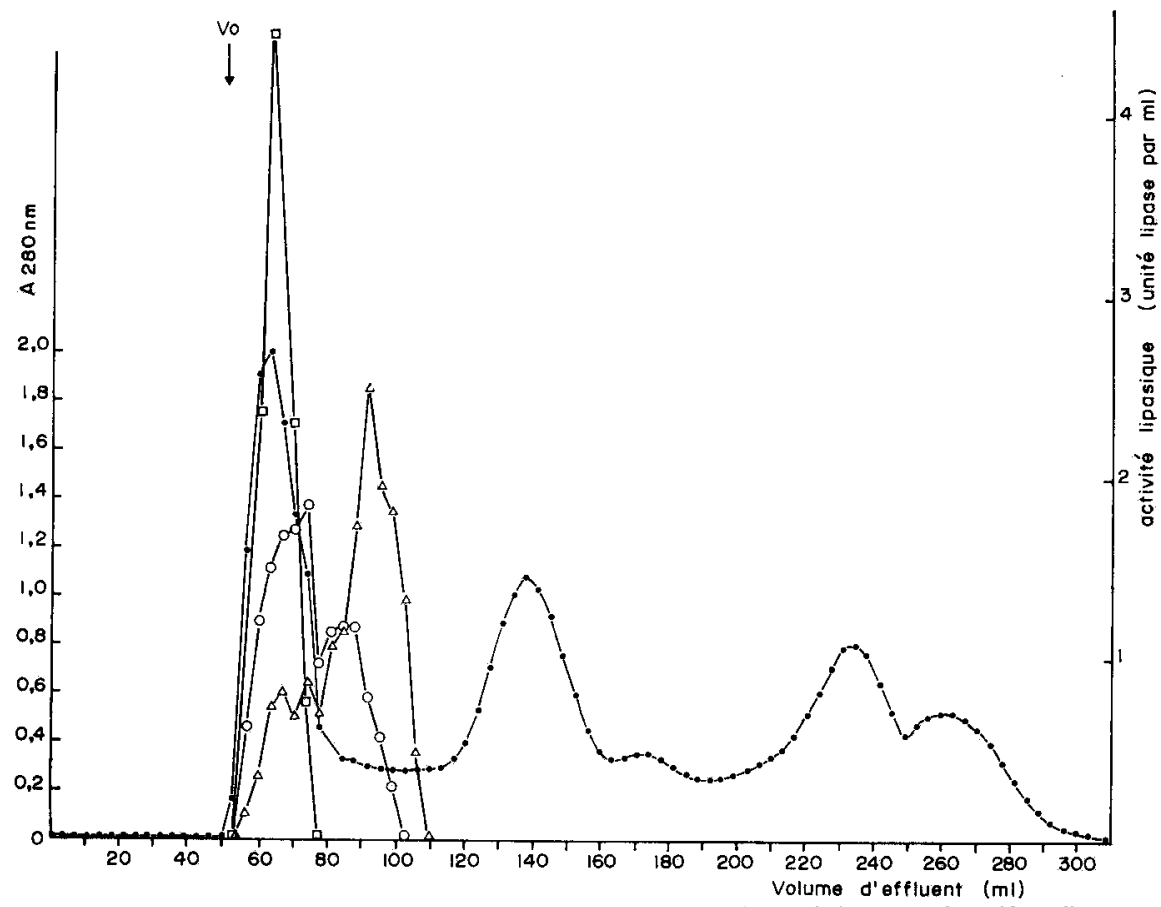

Fıg. 2. - Diagramme d'élution sur G 100 des protéines (.-....) précipitêes par le sulfate d'ammonium à la concentration $0,6 \mathrm{~S}$ (cf. texte). Ce diagramme reste identique quelle que soit la force ionique du milieu $(\mathrm{NaCl} 0, \mathrm{I} \mathrm{M} ; 0,25 \mathrm{M} ; 0,4 \mathrm{M})$.

Diagrammes d'élution de la lipase

dans $\mathrm{NaCl}$ o,I $\mathrm{M}: \square-\square-\square$, dans $\mathrm{NaCl} 0,25 \mathrm{M}: 0-0-0$, dans $\mathrm{NaCl} 0,4 \mathrm{M}: \Delta-\Delta-\Delta$.

associées pour constituer une certaine conformation de l'enzyme; dans ces deux cas, le sel ajouté au milieu, en modifiant notamment la constante diélectrique du solvant, pourrait rompre les interactions faibles, soit entre les agrégats et la lipase, soit entre les différentes sous-unités constitutives de l'enzyme. Il est à noter que l'augmentation de la force ionique du milieu ne modifie pas l'activité lipasique spécifique.

Reçu pour publication en décembre 1971.

\section{SUMMARY}

\section{IIPASE PURIFICATION TRIAL OF TROUT (SALMO GAIRDNERI RICH.) INTERCAECAL, TISSUE}

Purification includes the 3 following stages:

- preparation of a lyophilized powder from trout intercaecal tissue. The specific activity of this powder is 0.6 units lipase/mg of proteins ; 
- preparation of a defatted powder from the preceding samples;

- preparation of a lyophilized fraction from the preceding powder after extraction of the enzyme in a buffer medium, selective precipitation with ammonium sulfate and transit through Sephadex G Ioo resin. Specific activity is I5 units lipase/mg of proteins.

Shifting of the lipasic activity peak caused by the ionic force of the medium during transit through the resin, suggests some hypotheses on the lipase structure of this purification stage.

\section{RÉFÉRENCES BIBLIOGRAPHIQUES}

Brockerhoff H., I966. Digestion of fat by cod. J. Fish Res. Bd. Canada, 23, I835-1839.

Desnuelle P., Constantin M. J., Baldy J., I955. Technique potentiométrique pour la mesure de l'activité de la lipase pancréatique. Bull. Soc. Chim. Biol., 37, 285-290.

Laguesse E., 1894. Développement du pancréas chez les Poissons osseux. J. Anat. Physiol., 30, 79-II6.

Léger C., Bergot P., Flanzy J., François A.-C., r97o. Mise en évidence d'une activité lipasique dans le pancréas diffus de la Truite. Étude des modalités d'action de l'enzyme responsable. $C$. $R$. Acad. Sci. Paris, Série D, 270, 2813-2816.

Lowry O. H., Rosebrough N. J., Lewis Farr A., Randall R. J., I95x. Protein measurement with the Folin phenol reagent. J. biol. Chem., 183, $265-275$.

Verger R., De Hass G. H., Sarda L., Desnuelle P., I969. Purification from porcine pancreas of two molecular species with lipase activity. Biochim. biophys. Acta, 188, 272-282. 